\title{
Relativistic Hamiltonian Model for Higgs Resonance*
}

\author{
Charles B. Chiu, E. C. G. Sudarshan, and G. Bhamathi ${ }^{a}$ \\ Center for Particle and Department of Physics, University of Texas, Austin, Texas 78712, USA \\ a Department of Physics, University of Texas, Austin, Texas 78712, USA \\ North Texas Research Institute, University of North Texas, Denton, Texas 76203, USA
}

Z. Naturforsch. 52a, 140-142 (1997)

We construct a relativistic Hamiltonian model for Higgs resonance. Resonance trajectories are studied as a function of the parameters of the model. The possibility of a narrow width Higgs resonance in $1 \mathrm{TeV}$ region cannot be ruled out from general considerations. Our model may serve as a phenomenological guide in the search for Higgs resonance.

This is a brief report on our recent work [1]. The Higgs mechanism plays the crucial role in the unified electroweak theory. Through spontaneous symmetry breaking, the weak gauge bosons, $W$ and $Z$, acquire mass, while the photon remains massless. This explains why in the low energy region, weak interaction is much weaker than electromagnetic interaction. The Higgs mechanism also implies the existence of the Higgs particle. So far this particle has not been found.

In the minimal standard model, the mass, or more precisely the bare mass, of the Higgs particle is a free parameter. It is related to $\lambda_{0}$, the coupling in the quartic interaction, through the relation $m_{0}^{2}=2 \lambda_{0} v^{2}$, where $v$ is the vacuum expectation of the original Higgs field, which is about $1 / 4 \mathrm{TeV}$. We consider the scenario where the bare mass of the Higgs particle is very large, and in turn the coupling is strong. Here presumably the gauge interaction of the Higgs particle and its interaction with fermions are relatively unimportant.

Our main interest is the physics, say at the energy scale of $1 \mathrm{TeV}$. This energy scale is significantly above the masses of $W$ and $Z$. Here the interaction of Higgs particle with the gauge bosons is predominantly with the longitudinal components of the gauge bosons, which will be designated by the lower case symbols $w^{ \pm}, z$. These longitudinal components form an iso-

\footnotetext{
* Presented by Charles Chiu at a Workshop in honor of E. C. G. Sudarshan's contributions to Theoretical Physics, held at the University of Texas in Austin, September 15-17, 1991; “... My best wishes to George Sudarshan for many more years of inspirations to our scientific community."
}

Reprint requests to Prof. Dr. Charles B. Chiu. topic triplet of scalars. The interaction among this triplet is similar to the interaction among the isotriplet of pions. We recall, in the $\pi^{+} \pi^{-}$and $\pi^{0} \pi^{0}$ channels there is the $I=S=0, \sigma$ resonance. Similarly one may expect the presence of Higgs resonance in the $w^{+} w^{-}$ and $z z$ channels.

Various approaches have been used to study the occurrence of Higgs resonance in the $w^{+} w^{-}$and $z z$ channels. A partial list here includes [2]: the N/D method, the $1 / \mathrm{N}$ expansion in the $\mathrm{O}(2 \mathrm{~N})$ model, and various "unitarization schemes". For the latter, one finds that the Higgs resonance is present in the Padé approximation, and is absent in the $K$-matrix approach. We construct a Hamiltonian model for the $I=S=0$ eigenchannel, which is a linear combination of the $w^{+} w^{-}$and $z z$ channels. Our model is based on a relativistic hamiltonian theory [3]. Instead of the usual Hamiltonian, which specifies the energy of the system, we work with the "relativistic hamiltonian", which specifies the square of the center of mass energy. It is a Lorentz scalar. The matrix element of the free Hamiltonian is given by $H_{0}\left(s, s^{\prime}\right)=s \delta\left(s-s^{\prime}\right)$. Inspired by the separable potential model [4], we assume that the matrix element of the interaction term is factorizable, i.e. $H_{1}\left(s, s^{\prime}\right)=-g(s) g\left(s^{\prime}\right)$. Here a negative sign is appropriate for the present case, which corresponds to an attractive potential.

The solution of the eigenvalue equation of the full hamiltonian $H \phi=\left(H_{0}+H_{1}\right) \phi=\lambda \phi$ is given by

$$
\begin{aligned}
& \phi_{\lambda}(s)=\delta\left(s-s^{\prime}\right)-\frac{g_{\lambda} g_{s}}{\beta(s)} \cdot \frac{1}{\lambda-s+i \varepsilon}, \\
& \beta=1+\left\langle g^{2}\right\rangle,\left\langle g^{2}\right\rangle=\int \frac{g^{2}\left(s^{\prime}\right) \mathrm{d} s^{\prime}}{s-s^{\prime}+i \varepsilon} .
\end{aligned}
$$


Note that the wave function is a sum of the plane wave plus an outgoing spherical wave. The scattering amplitude is related to the coefficient of the outgoing spherical wave, In particular, at $s$ the amplitude is

$$
A(s)=\frac{\pi g^{2}}{\beta}=-\frac{\pi V}{1-\langle V\rangle},
$$

where in the last step the effective potential $V=H_{1}(s, s)$ $=-g^{2}(s)$ was introduced.

We assume that the effective potential is to be computed based on perturbation diagrams. More precisely, to avoid double counting, they are "s-channel two-particle irreducible diagrams". These diagrams up to one-loop level are shown in Figure 1. In our calculations we set the bare mass $m_{0} \rightarrow \infty$. For instance a $t$-channel tree diagram would correspond to $-2 \lambda_{0}\left[1+m_{0}^{2} /\left(t-m_{0}^{2}\right)\right] \rightarrow t / v^{2}$. At the tree level, the potential is given by $s /(16 \pi v)^{2}$. For the evaluation of the dispersion integral in the denominator function we adopt the minimal subtraction scheme, which amounts to removing all terms which have a polynomial dependence on the cutoff. The denominator function is then given by $\beta=\beta(s, v)$, where $v$ is an energy scale which is related to the subtraction constant. The solution $\beta(s, v)=0$ occurs on the second sheet of $s$. The trajectory of the resonance pole on the second sheet of the $W=\sqrt{s}$ plane, as a function of $v$, is shown in Fig. 2 as the dashed curve. Note that there is the trend of bending over near $\operatorname{Re} W=\sqrt{s}$ at $\sim 1.2 \mathrm{TeV}$. This implies an upper bound for the Higgs mass. The presence of this upper bound is qualitatively similar to the conclusions reached by earlier work [2].

For the result up to one-loop order, within our renormalization scheme the denominator function takes the form $\beta=\beta(s, v, R)$. For the minimal standard model, at resonance pole, it turns out that $R$ is a function of $v$. For this case, the trajectory of the resonance pole as a function of $v$ is displayed in Fig. 2 as the solid curve. The trend of this solid curve is similar to that of the dashed curve, except for an overall downward displacement. So for fixed $\operatorname{Re} W$, due to the additional loop contribution, there is an overall increase of the resonance width.

So far we have considered the Higgs resonance trajectory based on the minimal standard model. For the effective chiral lagrangian theory, the situation at the tree level is the same as that of the minimal standard model. At the one-loop level, the denominator function may again be written in the form of $\beta(s, v, R)$, except that $v$ and $R$ are now two independent parame- (a)

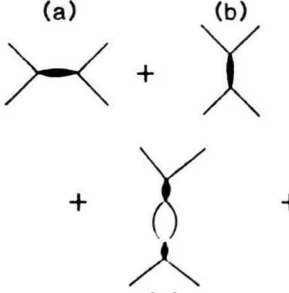

(d) (c)

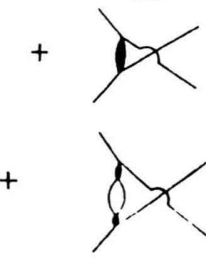

(e)
Fig. 1. Perturbation diagrams for the effective potential up to one-loop order.

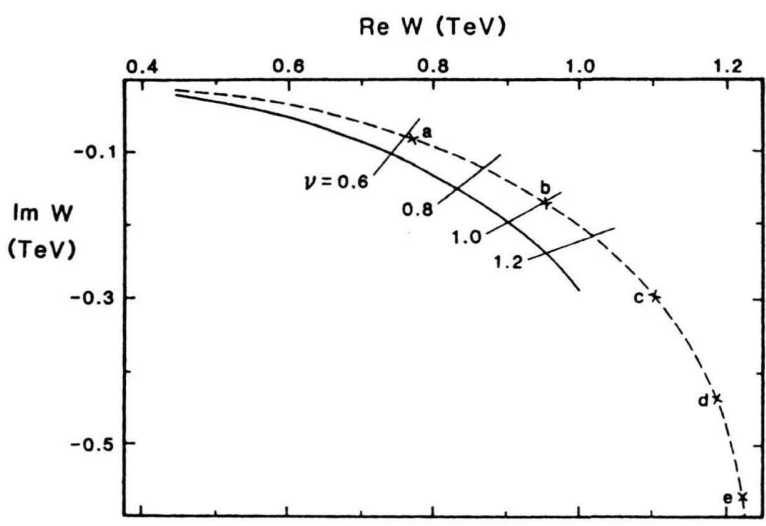

Fig. 2. Higgs resonance trajectories. Dashed curve: at tree level. Solid curve: up to 1-loop level in standard model.

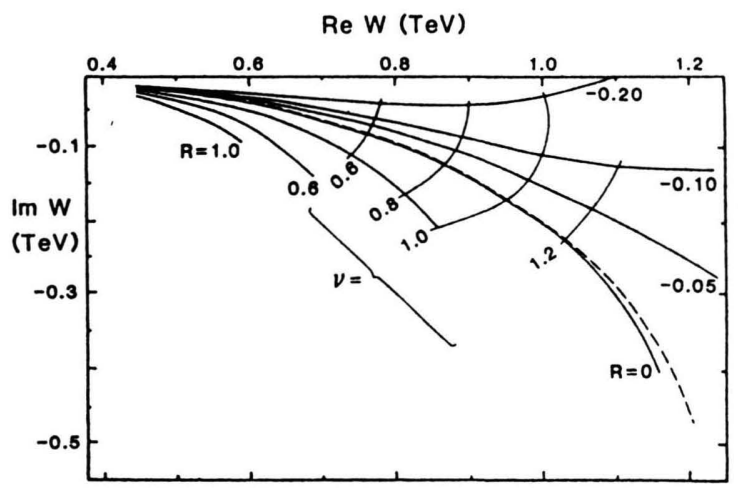

Fig. 3. The family of Higgs resonance trajectory up to 1-loop level based on the effective chiral lagrangian theory.

ters. Figure 3 illustrates the family of resonance trajectories for the present case. One interesting point here is that a narrow width of the Higgs resonance, say in $1 \mathrm{TeV}$ region, cannot be ruled out. 


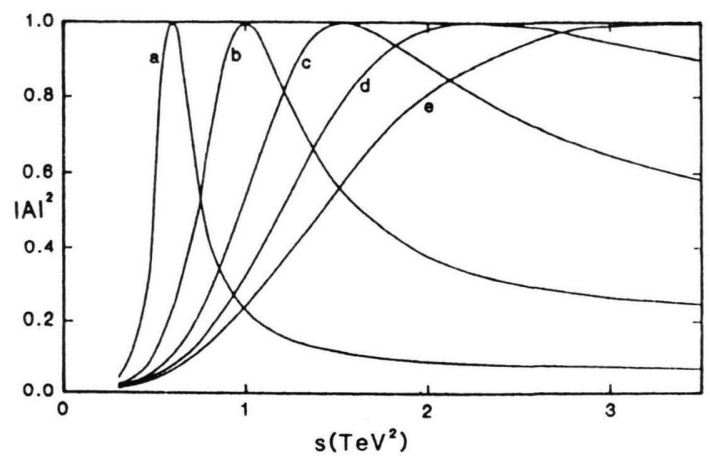

Fig. 4. Line shapes for those points along the dashed curve indicated in Fig. 2.

Finally we turn to the search of the Higgs resonance. We began by saying that in the minimal standard model, the bare mass of the Higgs particle is $a$ priori a free parameter. We are interested in the scenario where the bare mass is very large and the coupling is strong. Within our approach the Higgs reso-

[1] C. Chiu, E. C. G. Sudarshan, and G. Bhamathi, Hamiltonian model for Higgs resonance, CPT preprint, DOE259, 1991.

[2] See for examples: B. W. Lee, C. Quigg, and H. B. Thacker, Phys. Rev. D 16, 1519 (1977);

M. B. Einhorn, Nucl. Phys. B 246, 531 (1984);

Phys. Letters B211, 457 (1988); A. Dobado, M. J. Herrero, and Tran N. Truong, Phys. Letters B 235, 129, 134 (1990); Dobado, Phys. Lett. B 237, 457 (1990);

Dicus and Repko, Phys. Rev. D 42, 3660 (1990). nance is present over a wide range of parameter values. The position of the Higgs pole on the second sheet is governed by one or two parameters. Each choice of parameter(s) corresponds to a definite line shape in the cross section. Figure 4 shows sample line shapes for the tree level case, where the square of the normalized partial wave amplitude is plotted versus $s$.

Our model may be used as phenomenological guide in the search of the Higgs particle in the sub- $\mathrm{TeV}$ and $\mathrm{TeV}$ region. An optimistic scenario would be as follows. Through the interplay between model prediction and measurements, the data confirms one specific line shape of our model. This implies the discovery of the Higgs resonance, and at the same time it predicts the resonance pole position on the second sheet.

\section{Acknowledgement}

This work was supported by Department of Energy Grant No. DE-FG05-85ER40200.

[3] B. Bakamjian and L. H. Thomas, Phys. Rev. 92, 1300 (1953); E. C. G. Sudarshan, 1961 Brandeis Lectures. See more references in ref. 1.

[4] Y. Yamaguchi and Y. Yamaguchi, Phys. Rev. 95, 1628; 1635 (1954). 